\title{
Design and Comparison of Two-Loop with PI and Three-Loop Autopilot for Static Unstable Missile
}

\author{
Emad Mahrous Mohamed*, Lin Yan \\ School of Automation Science and Electrical Engineering, Beihang University BUAA), Beijing 100191, China. \\ * Corresponding author; Email: emadmahrous1977@yahoo.com \\ Manuscript submitted November 20, 2015; accepted February 10, 2016. \\ doi: 10.17706/ijcee.2016.8.1.1-11
}

\begin{abstract}
The characteristics of nonminimum phase and static unstable of a tail controlled tactical missile are presented firstly. Then, in order to eliminate the static error, a cascade PI compensator was introduced to the classic two loop autopilot. Due to the slow tracking for command acceleration, the longitudinal three-loop autopilot design is driven based on LTI model of missile plant to stabilize the nonminimum phase static unstably missile airframe. The focus is to explain the performance and the control effect at different values of velocity and stability derivative $\left(M_{\alpha}\right)$ of two algorithms on missile plant. The analysis is executed by establishing a standard algorithm in virtue of MATLAB/Simulink for autopilot design. The simulation results indicated that three-loop topology gives better tracking than two-loop with a cascade PI compensator at different value of stability derivative $M_{\boldsymbol{\alpha}}$. On the other hand, two-loop has a better response and less control effort at different velocities. fin angle and fin angle rate are less than the three loop for static unstable and stable missile.
\end{abstract}

Key words: PI compensator, two loop autopilot, three loop autopilot, flight control system, missile.

\section{Introduction}

An autopilot is a closed loop system, and it is the minor loop of the main guidance loop. The two- or three-loop autopilots have been introduced in tactical missiles in recent years [1], [2]. The lateral autopilots control missiles body by controlling surfaces to generate the required acceleration according to the guidance demand, such as; proportional navigation, augmented proportional navigation, line of sight, etc. In some Russia missile design, one accelerometer and one angular acceleration gyro are used and the accelerometer has to be positioned in the rear section of the missile for structure reasons. Nevertheless, the lateral autopilots with one accelerometer and one rate gyro are more commonly used in homing guidance tactical missiles [3]. The three-loop Raytheon has been designed especially for radar seeker missile to eliminate the coupling effect of radome and parasitic loop [4].

The classical two-loop autopilot consists of rate-damping loop which is used to act as damper and accelerometer loop which provides control of the lateral acceleration of the missile. But when adding a synthetic stability loop, it is called three-loop autopilot [5], [6]. Lateral autopilot acts as an inner loop of the guidance loop which is used to control the pitch and yaw motions. When the missile has two planes of symmetry, so we need consider one channel only, the pitch autopilot say. The structured autopilot design algorithm of flight path rate for tactical guided missile lateral autopilot has been presented [7], [8], where a design methodology has been developed by relevant analysis for a class of tactical guided missiles. The 
flight dynamic characteristics of the missile depend on its aerodynamic coefficients which vary significantly with flight condition such as altitude and Mach number. The problem is to design a pitch plane autopilot to track the normal acceleration commanded from the guidance system. The autopilot generates fin angle commands which are sent to the tail surface servos. By deflecting the tail fins, they generate aerodynamic forces and moments that maneuver the missile. Rate gyro and accelerometer measurements are processed by the flight control system to close the feedback control loop.

This paper is an elaborated comparison between two-loop with a cascade PI compensator and three loop autopilot. This paper is organized as follows. Section 2 describes the longitudinal autopilot model of a tailed controlled guided homing missile with one accelerometers and one rate gyro whereas the accelerometer is putted coincidence with center of gravity of the missile [9]. Section 3 presents two-loop autopilot design with a cascade PI compensator. A derivation of missile model needed for the three-loop autopilot design is given in Section 4. Section 5 presents the performance of the two algorithms. Section 6 introduces the conclusion of this paper.

\section{Autopilot and Missile Dynamic}

The longitudinal (vertical plane) flight control system for a bank to turn missile form a single input multioutput design model. The autopilot that will be designed will command normal body acceleration using tail fin deflection control. The plant outputs are normal acceleration $A_{z}\left(f t / s^{2}\right)$, and pitch rate $q(\mathrm{rad} / \mathrm{s}$ ), and the plant states are $\alpha, q, \delta$, and $\dot{\delta}$ (angle of attack, pitch rate, fin deflection, and fin rate respectively). The nominal longitudinal airframe dynamics is represented by $G(s)$. The deferential equation used to describe these open loop dynamic as in [10] are:

$$
\begin{aligned}
& \left.\begin{array}{l}
\dot{\alpha}=Z_{\alpha} \alpha+q+Z_{\delta} \delta_{e} \\
\dot{q}=M_{\alpha} \alpha+M_{\delta} \delta_{e}
\end{array}\right\} \\
& A_{z}=V Z_{\alpha} \alpha+V Z_{\delta} \delta
\end{aligned}
$$

Assuming that the actuator is second order system as

$$
\ddot{\delta_{e}}=-2 \zeta \omega \dot{\delta}_{e}-\omega^{2}\left(\delta_{e}-\delta_{c}\right)
$$

In the state space form, the airframe dynamics are represented by the following state space triple $(A, B, C)$ :

$$
\begin{gathered}
\dot{X}=A X+B U \\
Y=C X+D U \\
A\left[\begin{array}{cccc}
Z_{\alpha} & 1 & Z_{\delta} & 0 \\
M_{\alpha} & 0 & M_{\delta} & 0 \\
0 & 0 & 0 & 1 \\
0 & 0 & -\omega^{2} & -2 \zeta \omega^{2}
\end{array}\right], B=\left[\begin{array}{c}
0 \\
0 \\
0 \\
\omega^{2}
\end{array}\right], \\
C=\left[\begin{array}{cccc}
V Z_{\alpha} & 0 & V Z_{\delta} & 0 \\
0 & 1 & 0 & 0
\end{array}\right] .
\end{gathered}
$$

The transfer function matrix is $G(s)=C(s I-A)^{-1} B$. The longitudinal missile dynamics form a single input multioutput design model. From equation 4 , the transfer function matrix from the elevon fin 
deflection command $\delta_{c}$ to the normal acceleration $A_{z}$ and pitch rate $q$ is

$$
G(s)=\left[\begin{array}{c}
\frac{\omega^{2} V\left(Z_{\delta} s^{2}+Z_{\alpha} M_{\delta}-Z_{\delta} M_{\alpha}\right)}{\left(s^{2}-Z_{\alpha} s-M_{\alpha}\right)\left(s^{2}+2 \zeta \omega s+\omega^{2}\right)} \\
\frac{\omega^{2}\left(M_{\delta} s^{2}+M_{\alpha} Z_{\delta}-M_{\delta} Z_{\alpha}\right)}{\left(s^{2}-Z_{\alpha} s-M_{\alpha}\right)\left(s^{2}+2 \zeta \omega s+\omega^{2}\right)}
\end{array}\right]=\left[\begin{array}{l}
\frac{A_{z}(s)}{\delta_{c}(s)} \\
\frac{q(s)}{\delta_{c}(s)}
\end{array}\right]
$$

where $Z_{\alpha}, Z_{\delta}, M_{\alpha}, M_{\delta}$, and $M_{q}$ are the aerodynamic stability derivatives. The measurements that are available are normal acceleration $A_{z}=V Z_{\alpha} \alpha+V Z_{\delta} \delta(f t / s 2), q$ pitch rate (rad/s). The scalar control input $u=\delta_{c}$ (rad) is the fin angle command. The above aerodynamics have been linearized and represented a trim $\alpha$ angle of attack of 16 degrees, Mach number=0.8, $V=886.78$ (ft/s), an altitude of 4000 (ft.), actuator damping $\zeta=0.6$, and actuator natural frequency $\omega=113(\mathrm{rad} / \mathrm{s})$. The following parameters are the nominal values of the dimensional aerodynamic stability derivatives; $Z_{\alpha}=-1.3046(1 / \mathrm{s}) ; Z_{\delta}=$ $-0.2142(1 / \mathrm{s}) ; M_{\alpha}={ }_{-}^{+} 47.7109$ (1/s2) which were taken from [6]. The sign of $M_{\alpha}$ determines the stability of the open loop airframe. When the $M_{\alpha}$ is negative the airframe is stable, and when it is positive the airframe is unstable, which occurs when the aerodynamic center of pressure is forward of the center of gravity [5].

\section{Two-Loop Autopilot}

The two-loop autopilot system uses two loops to feedback an information of the missile motion to the forward path of the autopilot. One loop is involved with body rate information which is fed back using one rate gyro. The other is the missile acceleration, sensed using accelerometer that considered the main feedback loop. So, modelling of missile airframe dynamics is an important part of configuring an autopilot system. Missile dynamics is of nonlinear type. The two-loop autopilot structure and the missile dynamics transfer functions are displayed in Fig. 1. Equation (5) is used to design the normal acceleration command autopilot. In order to eliminate the static error, there are two controller blocks contained in the acceleration command autopilot. The control input is fin deflection command $\delta_{c}$ and the measured outputs are normal acceleration $A_{z}$ and pitch rate $q$. The longitudinal autopilot controller blocks $K_{A_{z}}(s)$ and $K_{q}(s)$ are designed to give a good acceleration command tracking and to ensure missile stability.

The controller blocks $K_{A_{z}}(s)$, and $K_{q}(s)$ consist of proportional-plus-integral (PI) control elements. The acceleration feedback loop controller block $K_{A_{z}}(s)$ has the structure

$$
K_{A_{z}}(s)=\frac{k_{z}\left(s+a_{z}\right)}{s}
$$

where the $k_{z}$ is the proportional gain, and $k_{z} a_{z}$ is the integral control gain. The pitch rate loop controller block $K_{q}(s)$ has the structure

$$
K_{q}(s)=\frac{k_{q}\left(s+a_{q}\right)}{s}
$$

The longitudinal autopilot design process is automated to vary acceleration feedback loop and pitch rate loop gains and evaluate longitudinal autopilot performance and robustness properties. The performance values examined are the normal acceleration command settling time, the percent undershoot, the percent overshoot and the steady state error. The two-loop autopilot feedback gains $k_{q}=-0.3, k_{z}=-0.00, a_{q}=$ 6 , $a_{z}=2$, and $a_{z}=2$. The contribution of the controller blocks $K_{A_{z}}(s)$, and $K_{q}(s)$ are analyzed in Section 4 . 




Fig. 1. Longitudinal autopilot.

\section{Three-Loop Autopilot}

The three loop autopilot is a new kind of autopilot developed recently years by one feedback loop (it is called synthetic loop) based on the traditional two loop autopilot. Also a pure integrator is contained in the forward path of the autopilot loop. The three loop pitch/yaw autopilot is used to most guided tactical missiles today as shown in Fig. 2. It has four gains $K_{D C}, K_{A}, K_{R}$, and $\omega_{I}$; which are used to control the third order dynamics of the autopilot. These dynamics are due to second order dynamics and an integrator that allows the flight control system to control unstable airframe. The airframe transfer functions for a tailed homing tactical missile are driven referring to (1), and (2) as following:

$$
\begin{gathered}
A_{z}=V Z_{\alpha} \alpha+V Z_{\delta} \delta_{e} \\
=V\left(\frac{Z_{\alpha}}{S} \dot{\alpha}+Z_{\delta} \delta_{e}\right) \\
=V\left(Z_{\delta} \delta_{e}+\frac{Z_{\alpha}}{S}\left(Z_{\alpha} \alpha+\frac{1}{s} \dot{q}+Z_{\delta} \delta_{e}\right)\right) \\
=V\left(Z_{\delta} \delta_{e}+\frac{Z_{\alpha}}{S}\left(Z_{\alpha} \alpha+\frac{1}{S}\left(M_{\alpha} \alpha+M_{\delta} \delta_{e}\right)+Z_{\delta} \delta_{e}\right)\right) \\
=V\left(\delta_{e}\left(Z_{\delta}+\frac{Z_{\alpha} Z_{\delta}}{S}+\frac{Z_{\alpha} M_{\delta}}{s^{2}}\right)+\frac{Z_{\alpha} \alpha}{s}\left(\frac{M_{\alpha}}{S}+Z_{\alpha}\right)\right) \\
=V\left(\delta_{e}\left(Z_{\delta}+\frac{Z_{\alpha} Z_{\delta}}{S}+\frac{Z_{\alpha} M_{\delta}}{s^{2}}\right)+\frac{1}{s}\left(\frac{A_{z}}{V}-Z_{\delta} \delta\right)\left(\frac{M_{\alpha}}{s}+Z_{\alpha}\right)\right) \\
=V\left(\delta_{e}\left(Z_{\delta}+\frac{Z_{\alpha} Z_{\delta}}{S}+\frac{Z_{\alpha} M_{\delta}}{s^{2}}-\frac{Z_{\delta}}{s}\left(\frac{M_{\alpha}}{s}+Z_{\alpha}\right)\right)-\frac{1}{s} \frac{A_{z}}{V}\left(\frac{M_{\alpha}}{S}+Z_{\alpha}\right)\right) \\
=V\left(\delta_{e}\left(Z_{\delta}+\frac{Z_{\alpha} M_{\delta}}{s^{2}}-\frac{Z_{\delta} M_{\alpha}}{s^{2}}\right)+\frac{1}{s} \frac{A_{z}}{V}\left(\frac{M_{\alpha}}{s}+Z_{\alpha}\right)\right.
\end{gathered}
$$

Collecting terms gives

$$
\begin{gathered}
\frac{A_{z}}{\delta_{e}}=\frac{V\left(Z_{\delta} s^{2}+Z_{\alpha} M_{\delta}-Z_{\delta} M_{\alpha}\right)}{\left(s^{2}-Z_{\alpha} s-M_{\alpha}\right)} \\
\frac{A_{z}}{\delta_{e}}=-\frac{V\left(Z_{\delta} M_{\alpha}-Z_{\alpha} M_{\delta}\right)}{M_{\alpha}}\left(\frac{1-\frac{Z_{\delta}}{Z_{\delta} M_{\alpha}-Z_{\alpha} M_{\delta}} s^{2}}{1+\frac{Z_{\alpha} s-\frac{s^{2}}{M_{\alpha}}}{M_{\alpha}}}\right) \\
\text { For } \Omega_{A F}=\sqrt{-M_{\alpha}}, \zeta_{A F}=\frac{Z_{\alpha} \Omega_{A F}}{2 M_{\alpha}} \\
K 1=-\frac{V\left(Z_{\delta} M_{\alpha}-Z_{\alpha} M_{\delta}\right)}{M_{\alpha}}, \Omega_{Z}^{2}=\frac{Z_{\delta} M_{\alpha}-Z_{\alpha} M_{\delta}}{Z_{\delta}},
\end{gathered}
$$

and for $A_{z}=n_{L}, q=\dot{\theta}$. Then, the airframe relationship between the fin angle and missile acceleration is given by

$$
\frac{A_{Z}}{\delta_{e}}=\frac{n_{L}}{\delta}=\frac{K 1\left(1-\frac{s^{2}}{\Omega_{Z}^{2}}\right)}{1+\frac{2 \zeta_{A F}}{\Omega_{A F}}-\frac{s^{2}}{\Omega_{A F}^{2}}}
$$


After then, it should be driven the transfer function between body rate $q$ and missile acceleration $A_{z}$. Refer to (1) and (2)

$$
\begin{gathered}
\quad q=\frac{1}{s}\left(M_{\alpha} \alpha+M_{\delta} \delta_{e}\right) \\
=\frac{1}{s}\left(M_{\alpha} \alpha+\frac{M_{\delta}}{Z_{\delta}}\left(\frac{A_{z}}{V}-Z_{\alpha} \alpha\right)\right) \\
=\frac{1}{s}\left(\left(M_{\alpha} \alpha-\frac{M_{\delta} Z_{\alpha}}{Z_{\delta}}\right) \alpha-\frac{M_{\delta}}{Z_{\delta}} \frac{A_{z}}{V}\right) \\
=\frac{1}{s}\left(\frac{\left(M_{\alpha}-\frac{M_{\delta} Z_{\alpha}}{Z_{\delta}}\right)\left(1+\frac{Z_{\delta}}{M_{\delta}} s\right)}{s-Z_{\alpha}+\frac{Z_{\delta} M_{\alpha}}{M_{\delta}}} q-\frac{M_{\delta}}{Z_{\delta}} \frac{A_{z}}{V}\right)
\end{gathered}
$$

Rearrange terms gives

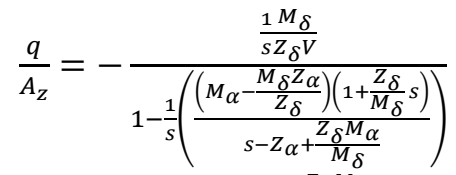

$$
\begin{aligned}
& =-\frac{M_{\delta}}{Z_{\delta} V} \frac{s-Z_{\alpha}+\frac{Z_{\delta} M_{\alpha}}{M_{\delta}}}{s^{2}+s\left(\frac{z_{\delta} M_{\alpha}}{M_{\delta}}-Z_{\alpha}\right)-\left(M_{\alpha}-\frac{M_{\delta} z_{\alpha}}{z_{\delta}}\right)\left(1+\frac{Z_{\delta}}{M_{\delta}} s\right)} \\
& =-\frac{M_{\delta}}{Z_{\delta} V} \frac{s+\frac{M_{\alpha} z_{\delta}-M_{\delta} z_{\alpha}}{M_{\delta}}}{s^{2}+s\left(\frac{z_{\delta} M_{\alpha}}{M_{\delta}}-Z_{\alpha}-\left(M_{\alpha} Z_{\delta}-M_{\delta} Z_{\alpha}\right) \frac{1}{M_{\delta}}\right)-\left(M_{\alpha}+\frac{M_{\delta} Z_{\alpha}}{Z_{\delta}} s\right)} \\
& =-\frac{M_{\delta}}{Z_{\delta} V} \frac{s+\frac{M_{\alpha} z_{\delta}-M_{\delta} z_{\alpha}}{M_{\delta}}}{s^{2}-\frac{M_{\alpha} Z_{\delta}-M_{\delta} z_{\alpha}}{Z_{\delta}}} \\
& =\frac{1}{V} \frac{1+\frac{M_{\delta}}{M_{\alpha} Z_{\delta}-M_{\delta} Z_{\alpha}} s}{1-\frac{Z_{\delta}}{M_{\alpha} Z_{\delta}^{-M_{\delta} z_{\alpha}}} s^{2}}
\end{aligned}
$$

By introducing a new variable $T_{\alpha}=\frac{M_{\delta}}{M_{\alpha} Z_{\delta}-M_{\delta} Z_{\alpha}}$ then

$$
\frac{q}{A_{z}}=\frac{\dot{\theta}}{n_{L}}=\frac{1}{V} \frac{1+T_{\alpha} S}{1-\frac{s^{2}}{\Omega_{Z}^{2}}}
$$

And the transfer function that relating between missile body rate $q$ to the fin angle $\delta_{e}$ is given by

$$
\begin{gathered}
\frac{q}{\delta_{e}}=\frac{A_{z}}{\delta_{e}} \frac{q}{A_{z}}=\frac{K 1\left(1-\frac{s^{2}}{\Omega_{Z}^{2}}\right)}{1+\frac{2 \zeta_{A F}}{\Omega_{A F}}-\frac{s^{2}}{\Omega_{A F}^{2}}} \frac{1}{V} \frac{1+T_{\alpha} s}{1-\frac{s^{2}}{\Omega_{Z}^{2}}} \\
=\frac{K 3\left(1+T_{\alpha} s\right)}{1+\frac{2 \zeta_{A F}}{\Omega_{A F}-\frac{s^{2}}{\Omega_{A F}^{2}}}}
\end{gathered}
$$

where $3=\frac{K 1}{V}$. For the three loop autopilot, it includes an integrator for body rate in order to reduce the steady state error. It should be clear from Fig. 2 that the acceleration feedback loop is a proportional controller acting on the acceleration error. The inner loops form a proportional plus integral (PI) for pitch rate to stabilize the missile body. The outer loop relationship is given by

$$
e=A_{z C} K_{D C}-A_{z}
$$

where $A_{z}$ is the measured output acceleration and $A_{z c}$ is the input acceleration command. 


$$
\frac{A_{z}}{A_{z c}}=K_{D C} \frac{G(s) G_{1}(s)}{1+G(s) G_{1}(s)}
$$

where $G_{1}(s)=\frac{A_{z}}{\delta_{e}}, G_{2}(s)=\frac{q}{\delta_{e}}$, and

$$
G(s)=\frac{\delta_{e}}{e} K_{R}\left(\frac{\omega_{I}}{s}\left(G_{2}(s) \delta-e K_{A}\right)+G_{2}(s) \delta\right)=\frac{1}{s} \frac{G_{M}(s) K_{R} \omega_{I} K_{A}}{K_{R} G_{M}(s) G_{2}(s)\left(\frac{\omega_{I}}{s}+1\right)-1}
$$

with substitution from (8) and (12) into equation (14), the final result is

$$
\frac{A_{z}}{A_{z c}}=K_{D C} \frac{G_{M}(s) K K 1\left(1-\frac{s^{2}}{\Omega_{Z}^{2}}\right)}{\left\{\begin{array}{c}
G_{M}(s) K_{o}+\left[K_{R} G_{M}(s)\left(1+\omega_{I} T_{\alpha}\right)-1\right] s \\
\left.+\left[G_{M}(s)\left(\left(K_{R} K 3 T_{\alpha}-\frac{K K 1}{\Omega_{Z}^{2}}\right)\right)-\frac{2 \zeta_{A F}}{\Omega_{A F}}\right] s^{2}-\frac{s^{3}}{\Omega_{A F}^{2}}\right\}
\end{array}\right.}
$$

where $K=K_{R} \omega_{I} K_{A}, K_{o}=K\left[\left(\frac{K 3}{K_{A}}\right)+K 1\right]$.

For common practice it is assume that the actuator dynamic are very fast relative to the system response $G_{M}(s) \cong 1$. The closed loop transfer function after approximation is

$$
\frac{A_{z}}{A_{z c}}=K_{D C} \frac{K K 1\left(1-\frac{s^{2}}{\Omega_{Z}^{2}}\right) / K_{o}}{\left\{+\left[G_{M}(s)\left(\left(K_{R} K 3 T_{\alpha}-\frac{K K 1}{\Omega_{Z}^{2}}\right)\right)-\frac{2 \zeta_{A F}}{\Omega_{A F}}\right] s^{2} / K_{o}-\frac{s^{3}}{K_{O} \Omega_{A F}^{2}}\right\}}
$$

Finally, for zero steady state error to a step input, it is required that the closed loop gain is

$$
\frac{K_{D C} K K 1}{K_{O}}=1
$$

The longitudinal autopilot design process is automated to vary the acceleration feedback loop and the pitch rate loop gains and evaluate longitudinal autopilot performance and robustness properties. The performance values examined are the normal acceleration command settling time, the percent undershoot, the percent overshoot and the steady state error. It found that the three-loop autopilot feedback gains are $k_{D C}=1.061, k_{A}=-0.007, \omega_{I}=18.8, k_{R}=-0.7$. The contribution of the three loop gains as shown in Fig. 3 , Fig. 5, and Fig. 6 but it gives a nonzero error as shown in Fig. 4 at different velocity because $K 1$ is dependent on the missile velocity $V$.



Fig. 2. Standard three-loop autopilot as in [11]. 


\section{Results and Discussion}

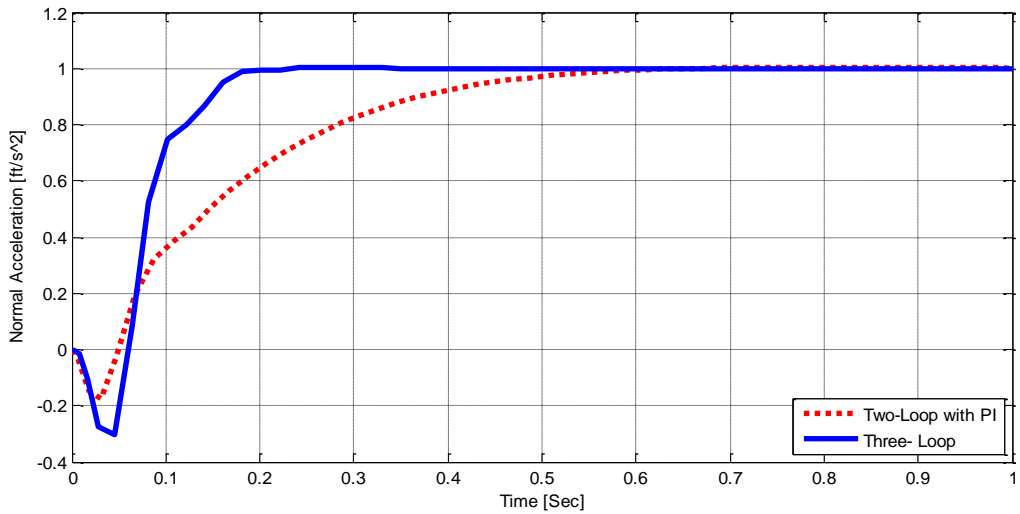

(a)

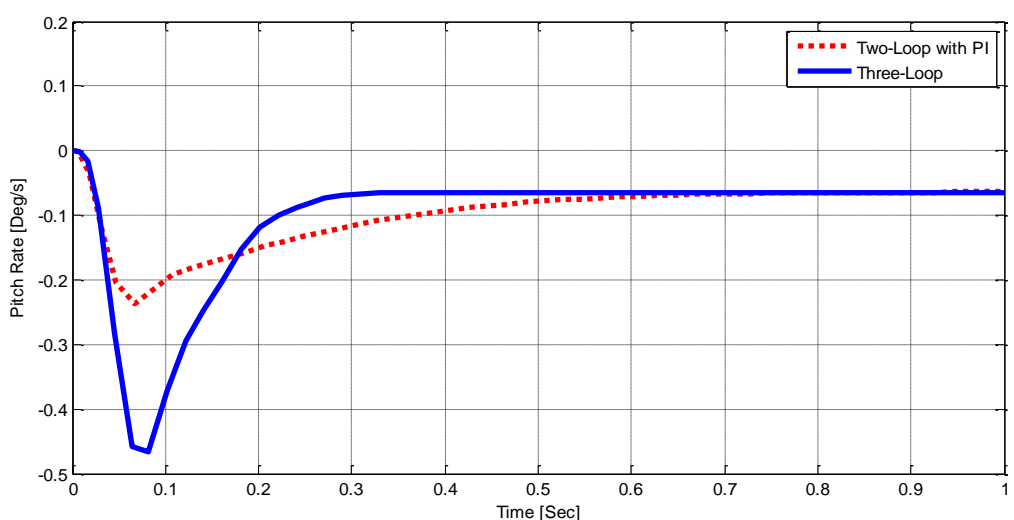

(b)

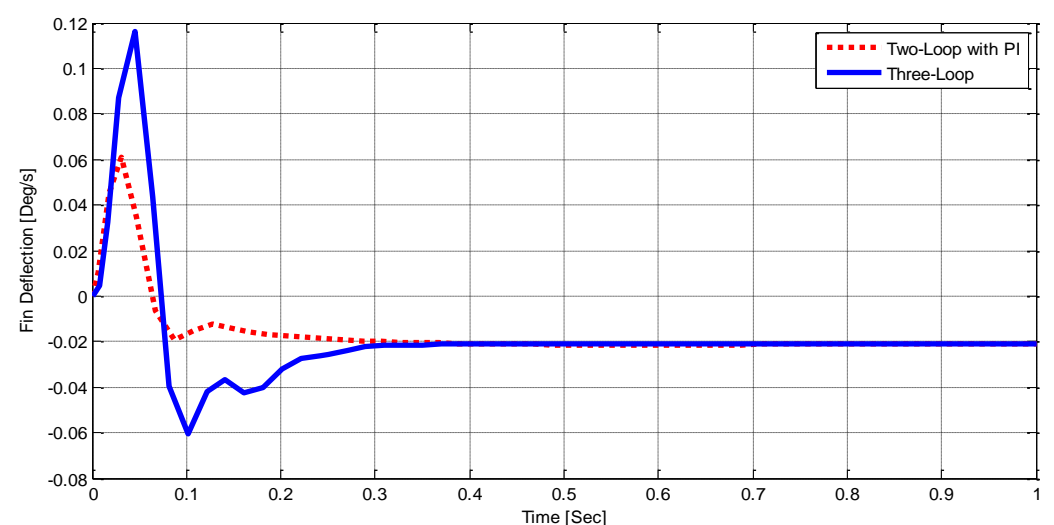

(c)

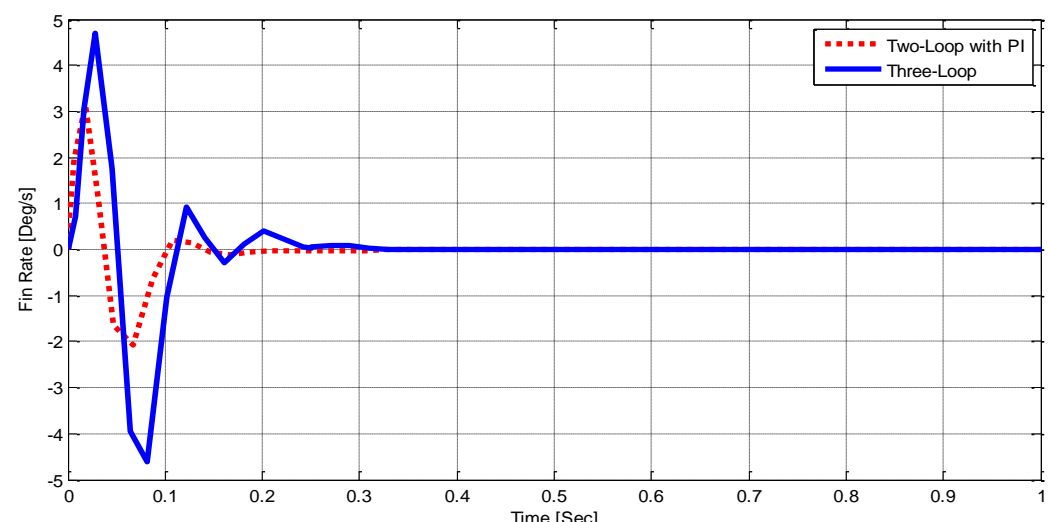

(d)

Fig. 3. Unit step response history of two topologies. 
Note that the aerodynamics acceleration transfer function contains a right half plane (RHP) zero. This no minimum phase relationship results from the missile fin deflection initially producing a lift force in the direction opposite to the command. The moment due to fin force cause the airframe to pitch, creating an acceleration command. This phenomena is observed in the normal acceleration response as shown in Fig. 3-5 as an initial undershoot in the time history of normal acceleration, because the initial value of step response is

$$
\lim _{s \rightarrow \infty} s\left[\frac{1}{s} \frac{A_{z}(s)}{\delta_{e}(s)}\right]=Z_{\delta}<0
$$

But the final value of the step response is

$$
\lim _{s \rightarrow 0} s\left[\frac{1}{s} \frac{A_{z}(s)}{\delta_{e}(s)}\right]=\frac{Z_{\alpha} M_{\delta}-M_{\alpha} Z_{\delta}}{-M_{\alpha}}>0
$$

and the stability of the airframe is governed by the value of the stability derivative $M_{\alpha}$. when $M_{\alpha}$ is negative, the airframe is stable and when $M_{\alpha}$ is positive the airframe is unstable.

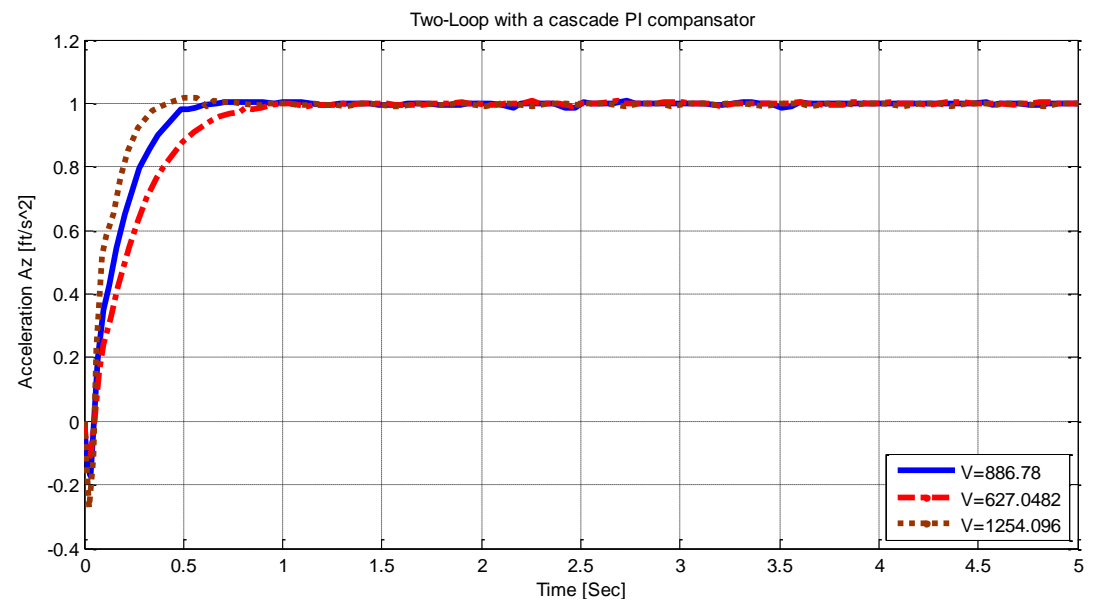

(a)

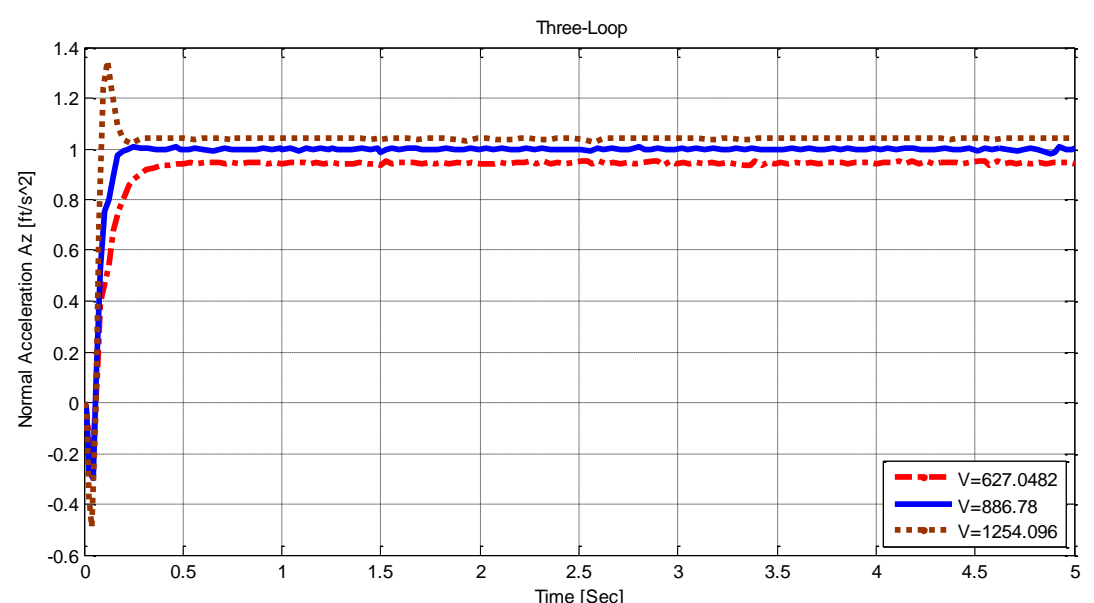

(b)

Fig. 4. Normal Acceleration $A_{z}$ of two-loop and three-loop at different value of velocity.

It is seen from Fig. 4 that for different value of velocity $886.78 \sqrt{2}, 886.78$ and $886.78 / \sqrt{2}$ (ft/s) respectively, two-loop has better response than three-loop that it has a steady state error and big overshoot 
when the velocity is changed. The analysis results as shown in Fig. 5 at different value of $M_{\alpha}$ show that the three-loop classic autopilot has the best robustness properties. In Fig. 6 it is clear that the control input of two-loop with a cascade PI compensator is half value for all different value of $M_{\alpha}$ compared with the control input of three-loop topology.

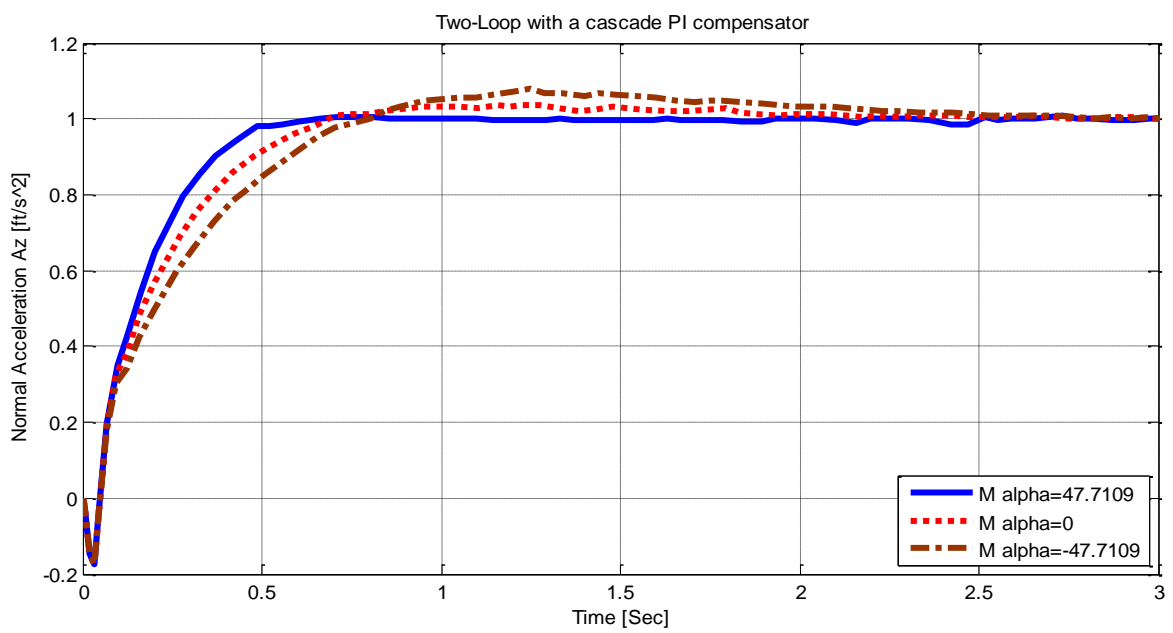

(a)

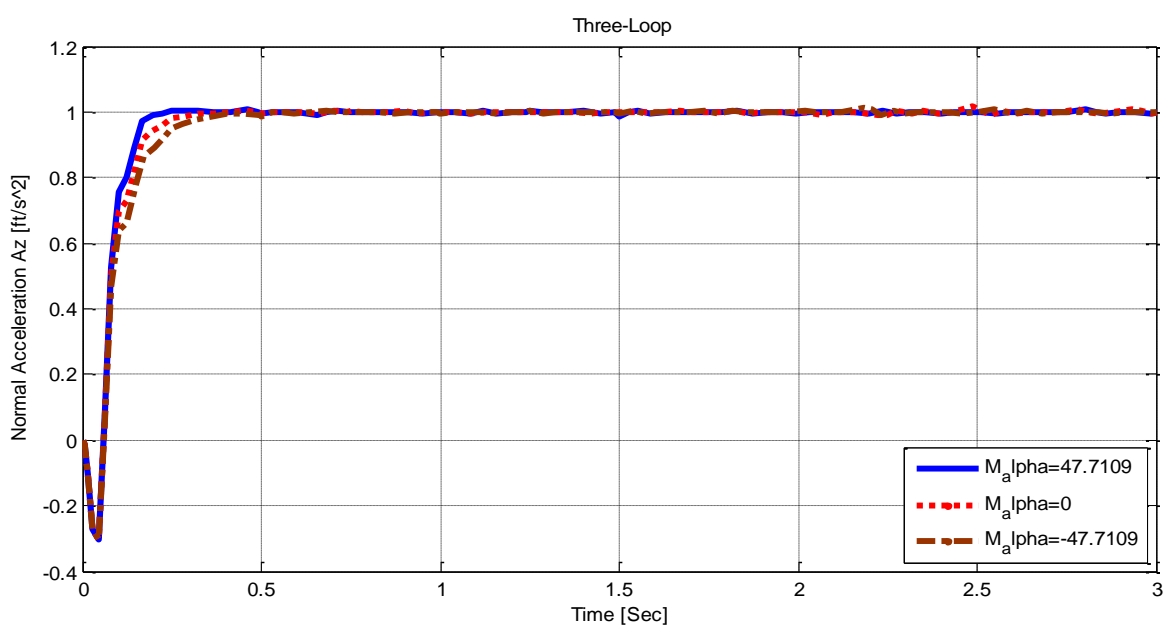

(b)

Fig. 5. Normal acceleration $A_{z}$ of two-loop and three-loop at different value of $M_{\alpha}$.



(a) 


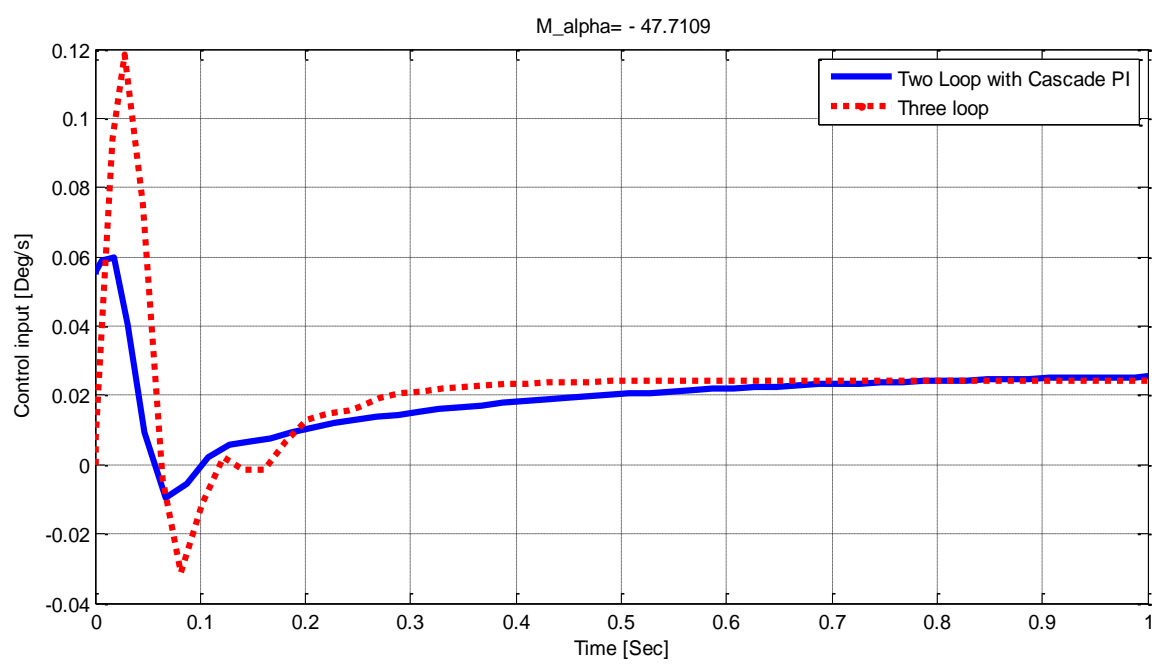

(b)

Fig. 6. Control input step response of two topology at different value of $M_{\alpha}$.

\section{Conclusion}

Three-loop topology is faster than two loop for tracking normal acceleration. In addition, it is more robustly than two-loop for different value of aerodynamic stability derivative $M_{\alpha}$ which it occurs due to movement of the center of gravity because of fuel burn during boost and changes in the aerodynamic conditions during glide flight. However, two-loop with a cascade PI controller has a three advantages better than three loop as follows: firstly, it has better response at different velocities because the PI compensator eliminates the static error of the system. Secondly, the control input and the fin deflection have half values compared to three loop at different values of stability derivative $\left(M_{\alpha}\right)$ which introduces negative value for stable missile and positive value for unstable missile. Finally, it has a small value of the fin deflection, fin rate, and pitch rate which could produce moderate actuator rates without rate saturation for sudden pitch rate demands. In future, to design a high-performance autopilot for the whole entire missile flight envelop, it is convenient to use a robust or adaptive control techniques.

\section{References}

[1] Paul, Z. (2002). Tactical and Strategic Missile Guidance. Virginia: AIAA Inc., 70-73.

[2] Arrow, A., \& Williams, D. E. (1987). Comparison of classical and modern autopilot design and analysis techniques for a tactical air-to-air bank-to-turn missile.

[3] Devaud, E., \& Siguerddidjane, H. F. S. (2000). Some control strategies for high-angle-of-attack missile autopilot. Control Engineering practice, 8, 885-892.

[4] Garnel, P. (2003). Guided Weapon control systems. Beijing Institute of Technology.

[5] Nesline, F. W., \& Nesline, M. L. (1984). How autopilot requirements constrain the aerodynamic design of homing missiles. Proceedings of the Conference on American Control.

[6] Fan, L. F., Chen, Y., \& Lin, P. (2014). Control analysis for a non-minimum phase static unstably missile. Proceedings of the 14th International Conference on Control, Automation and Systems (pp. 947-952).

[7] Das, G., Datta, K., Ghoshal, T. K., \& Goswami, S. K. (1996). Structured designed methodology of missile autopilot. Journal of the Institution of Engineers (India), 76, 49-59.

[8] Das, G., Datta, K., Ghoshal, T. K., \& Goswami, S. K. (1998). Structured designed methodology of missile autopilot - II. Journal of the Institution of Engineers (India), 79, 28-34.

[9] Wise, K. A. (2008). Robust stability analysis of adaptive missile autopilots. Proceedings of the AIAA 
Guidance on Navigation and Control conf.

[10] Wise, K. A., \& Eberhardt, R. (1992). Automated gain schedules for missile autopilots using robustness theory. Proceedings of the First IEEE Conference on Control Applications (pp. 243-250).

[11] Zarchan, P. (1997). Tactical and strategic missile guidance. Progress in Astronautics and Aeronautics, 176.

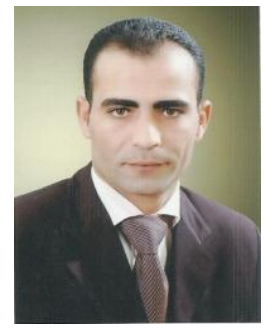

Emad Mahrous Rabie Mohamed was born in 1977. He received his B.S degree in electrical power engineering from Military Technical College, Cairo, Egypt in 2002 and the M.S. degree in control system from Cairo University, Egypt in 2009. He is currently a Ph.D. student in the Department of Automatic Control, School of Automation Science and Electrical Engineering, Beihang University BUAA), Beijing, China. He started in 2014 for his Ph.D. degree. His main research interest is missile guidance and control systems.



Yan Lin was born in 1955. He received the M.S. and Ph.D. degrees from Beihang University (BUAA), in 1988 and 1999, respectively. He is currently a professor in the School of Automation, BUAA. His research interests include robust control and adaptive control. Prof. Lin is in charge of National Natural Science Foundation of China, Doctoral Fund, Beijing Natural Science Foundation and Sub-Topic of 863 Project, and participates in project of Beijing key Discipline Foundation. As a major participant of National Natural Science Foundation of China, he won the second prize of 1998 Science and Technology Progress Award of Aviation Industry Corporation. He is a coauthor of more than 50 papers published in the IEEE and other international journals. 